\section{LA SCULPTURE AFRICAINE DANS LES ECRITS SUR L'ART D'ANDRÉ MALRAUX} Jean-Pierre Zarader
Cette logique qui lie la répétition à l'altérité.

Jacques Derrida

La métamorphose que subit le masque lorsqu'il entre au musée efface la sorcellerie, n'efface pas son empreinte.

André Malraux

Ce qui se joue à propos de l'art africain, et notamment des fétiches qui occupent une si grande place dans les Ecrits sur l'art, c'est la tension - la relation agonistique - entre formes et forces. Dans l'annexion des œuvres réfractaires - au premier rang desquels les fétiches -, c'est l'identité du Musée Imaginaire qui est en jeu et c'est la notion même de métamorphose qui est interrogée. C'est ce qu'avait bien perçu Picasso, et c'est aussi ce que Braque, selon lui, n'avait pas perçu (aux dires de Jacqueline rapportés par Malraux dans La Tête d'Obsidienne):* "Picasso seul, lorsqu'il découvrit le Musée du Trocadéro, ressentit leur caractère magique, indifférent à ses amis."* Picasso lui-même, dans le dialogue imaginaire de $L a$ Tête d'Obsidienne, récusant le rapprochement si souvent établi $-\mathrm{y}$ compris par Malraux ${ }^{1}$ - entre cubisme et sculpture africaine, insiste sur cette distinction:

On parle toujours de l'influence des Nègres sur moi. Comment faire? [...] Leurs forme n'ont pas eu plus d'influence sur moi que sur Matisse. Ou Derain. Mais pour eux, les masques étaient des sculptures comme les autres [...] Les masques, ils n'étaient pas des sculptures comme les autres. Pas du tout. Ils étaient des choses magiques [...] ils étaient des intercesseurs, je sais le mot en français depuis ce temps-là. Contre tout; contre les esprits inconnus, menaçants. Je regardais toujours les fétiches. J'ai compris: moi aussi je suis contre tout.*

Quand on sait l'importance du "Non" chez Malraux - du refus d'Antigone à l'art comme anti-destin -, et son souci constant d'interroger le mystère, on mesure l'intérêt de ces quelques lignes

\footnotetext{
1 "L'anguleuse parenté du cubisme avec l'art nègre est manifeste" (L'Intemporel: 259).
}

* (MALRAUX, André. La Tête d'Obsidienne. Paris: Gallimard, 1974: 20. Traduction brésilienne à paraître, Edson Rosa da Silva.)

* (MALRAUX, André. L'Intemporel. Paris: Gallimard, 1976: 244.)

(MALRAUX, André. La Tête d'Obsidienne: 17-18.) 
* (MALRAuX, André. L'Intemporel: 300.$)$

* (Ibidem: 362.) et l'affinité profonde qui unit Malraux, Picasso et la sculpture africaine. Ce n'est pas un hasard si Picasso en arrive, dans certains tableaux (Chapeau bleu, Enfant à la langouste, Femme à l'artichaut), “œuvres limites" annonçant les Tarots, à évoquer ce que Malraux nomme un "Ordre de la distorsion".* L'expression exprime à elle seule cette tension agonistique héritée des sculpteurs africains et que l'on retrouve dans les dernières toiles de Picasso. Ce qui ne renvoie pas simplement, comme on est souvent tenté de l'affirmer un peu vite, à un affrontement plus ou moins nietzschéen entre l'apollinien et le dionysiaque: cet "Ordre de la distorsion" dit à la fois l'harmonie et la destruction de l'harmonie, la forme et la force - voire la violence - qui tend à la détruire, mais qui ne peut véritablement tendre à la détruire, artistiquement, ${ }^{2}$ qu'en devenant elle-même forme. Comprenons bien la question qui se pose ici: peut-on parler d'une "relève" (Aufhebung) au sens que Hegel donne à ce terme, c'est-à-dire, pour le dire un peu vite, d'une négation qui serait en même temps un dépassement et un achèvement? On peut, bien sûr être tenté de le faire et certains textes malruciens vont manifestement dans ce sens puisque "tout finit au Musée Imaginaire"* et que, comme aime à le répéter Malraux, "à la fin, c'est toujours la métamorphose qui gagne".* Mais on peut aussi, et contradictoirement, affirmer que ce qui - dans les textes consacrés aux fétiches - tente de se dire est l'impossibilité de toute "relève", la perte de l'harmonie et l'impossibilité d'une belle totalité - ce qui inscrit Malraux, et de manière radicale, dans la pensée contemporaine.

$\mathrm{Au}$ fond, c'est le devenir forme d'une force - la métamorphose de la force en forme - que soulignent l'art africain et les Tarots de Picasso. Mais comme cette formule vaudrait pour toute œuvre d'art et pour toute création, il faut ajouter que ce devenir-forme, cette métamorphose, est inachevée et inachevable. Non pas, ou non pas seulement, parce que toute métamorphose est soumise à l'histoire et que l'histoire de l'art et des métamorphoses comme toute histoire est révisionniste et s'écrit au présent: "Entre Corneille et

${ }^{2}$ La précision est bien sûr essentielle: toute création est rupture et révolte (que ce soit contre la Création ou contre les formes héritées des grands maittres, voire, comme chez Picasso, contre ses propres formes et son propre style), mais elle se fait au bénéfice de l'art: Breton, comme Lautréamont, "finira dans la Pléiade”. Quant à la révolte contre l'art, qui loin de nourrir l'art, n'est qu'une négation de l'art, Malraux l'a stigmatisée dans La Tête d'Obsidienne (pp. 75-80). La révolte d'un artiste, aussi radicale soit-elle, reste une révolte d'artiste, au sens où Malraux parle d'une biographie d'artiste - une "faculté transformatrice." (Les Voix du silence: 418 .) 
Campistron, le cortège de nos gloires - puisque nous avons oublié celles de leur temps - est celui des recalés".* Les "recalés", ce sont ces oubliés de l'histoire et de l'histoire de l'art que la métamorphose, dans une actualisation du passé, ressuscite. Cette affirmation de Malraux, portant sur la littérature, est intéressante car elle s'applique, mutatis mutandis, à la sculpture africaine. Celle-ci relève bien en un sens de cette "tradition des vaincus", chère à Walter Benjamin, et que Malraux retrouve lorsqu'il évoque les "recalés". Et si Malraux dénonce à juste titre la longue mise à l'écart de cet art qui n'était pas reconnu comme tel: "La sculpture africaine resta longtemps prisonnière des collections d'ethnographie" $3,{ }^{*}$ il aurait pu reprendre à son compte - comme le fait à sa manière le film d'Alain Resnais et de Chris Marker, Les statues meurent aussi - l'affirmation de Benjamin qui se donne pour tâche de "brosser l'histoire à rebrousse-poil":

L'identification au vainqueur bénéficie toujours aux maitres du moment [...] Le butin, selon l'usage toujours, est porté dans le cortège. C'est ce qu'on appelle les biens culturels [...] Car il n'est pas de témoignage de culture qui ne soit en même temps un témoignage de barbarie.*

Mais cette approche politique du regard de Malraux - et d'autres auteurs, que ce soit Carl Einstein ou Chris Marker - sur la sculpture africaine mériterait une étude spécifique, et je m’en tiendrai donc ici à une approche davantage conceptuelle qui tente de saisir la place de cette sculpture dans les Ecrits sur l'art et son rapport aux concepts malruciens comme ceux de métamorphose ou de Musée Imaginaire. Ici, dans le cas des fétiches, comme dans d'autres cas exceptionnels (Les Goya, le Shigemori de Takanobu), si la métamorphose est inachevable c'est parce qu'elle ne saurait se faire sans reste - ce qui marque bien l'impossibilité d'une "relève" du sacré dans l'esthétique, d'une totalité achevée: ce reste c'est, littéralement, ce que Malraux nomme l'empreinte ou l'aura.

Mais peut-être faudrait-il commencer par le commencement, et se poser la question: pourquoi Malraux s'intéresse-t-il à la sculpture africaine? Il y a l'époque, bien sûr: Malraux n’a été ni le premier, ni le seul, à s'intéresser à l'art africain et notamment à la sculpture africaine. Dès le début du XX $\mathrm{X}^{\circ}$ siècle (le livre de Carl

\footnotetext{
${ }^{3}$ La formule est frappante : elle ne constate pas simplement un fait, elle contient en puissance toutes les tensions qui peuvent exister - et qui n'ont pas manqué d'exister - entre ethnologie et esthétique.
}

* (MALRAUX, André. L'Homme précaire et la littérature. Paris, Gallimard, 1977: 257.)

* (MAlRAuX, André. L'Intemporel: 244.)

* (BENJAMIN, Walter. "Sur le concept d'histoire". In: CEuvres III. Paris: Gallimard, "folio-essais», 2000: 432433.) 
* (JAMIN, Jean: "L'Afrique en tête Le jazz et La création du monde". In: JAMIN, Jean et WILLIAMS, Patrick, Une anthropologie du Jazz. Paris: CNRS Editions, 2010: 292.)

* (MALRAUX, André. L'Intemporel: 270.)
Einstein, Negerplastik, paraît en 1915 - et sera traduit en français en 1961, par Jean Laude, et en 1998 par Liliane Meffre -, les textes d'Apollinaire sont de la même période, ceux de Breton plus tardifs) les écrivains et plus généralement les artistes ont pressenti que quelque chose d'essentiel allait se jouer là, dans cette rencontre de l'Occident et de cet art qui apparaissait à la fois primitif, sauvage et étrangement proche. On pourrait donc dire que le siècle s'ouvre sur cette affinité entre les écrivains européens et ces étranges objets sans nom ${ }^{4}$ venus d'ailleurs. La voie est ouverte dès 1912, date de la reprise théâtrale des Impressions d'Afrique de Raymond Roussel, représentation à laquelle assistaient Guillaume Apollinaire, Francis Picabia, Marcel Duchamp. Le primitivisme qui naît à cette époque voit dans l'Afrique - dans sa musique comme dans sa sculpture - une source de vie, d'authenticité et d'altérité originelle susceptible de régénérer la civilisation occidentale, comme l'explique très bien Jean Jamin:

[...] devait s'ensuivre non seulement un renouveau de l'activité poétique mais un retour de l'art dans la vie, du rêve dans la pensée, du geste dans le langage, de l'imaginaire dans le réel, de la magie dans l'objet... [...] Il s'agissait de restaurer un rapport sensible et sensuel entre l'homme et la nature, rapport que le "penser dirigé" (Tzara) de la civilisation occidentale [...] avait distendu, brisé et vicié.*

Or Malraux, en plus d'un sens, est à l'opposé d'une telle pensée. Il n'a cessé de souligner la nécessité de distinguer l'art et la vie, l'imaginaire et le réel ${ }^{5}$ - et il est plus que réticent à faire l'éloge de la sauvagerie: “On étudie 'l'ensauvagemen' de notre sensibilité. Il ne me semble pas aller de soi. L'un des pôles de la sauvagerie est l'informe, peut-être l'éphémère [...] Ce n'est pas 'le plus barbare' qui nous retient, c'est la plus enfoui, le plus profond. Ce n'est pas dans le plus précaire, dans la figure de paille, que s'approfondit la sauvagerie". ${ }^{* *}$ Malraux va donc s'appliquer - et on voit bien là son

4 "Si bien que les jeunes artistes trouvèrent en eux [les fétiches] des objets sans précédent et sans nom, car on ne les nommait pas encore objets d'art." (MALRAUX, A. L'Intemporel: 244).

${ }^{5}$ C'est pourquoi Malraux s'appliquera à distinguer imaginaire et imagination: "L'imagination est un domaine de rêves, l'imaginaire, un domaine de forme" (L'Homme précaire et la littérature: 179). Ainsi le Musée Imaginaire, tel que le conçoit Malraux, est-il proprement un Musée de l'Imaginaire.

${ }^{6}$ A la figure de paille Malraux opposera «les sculptures vaudou demeurées au Dahomey». Cette même référence - accompagnée de sa reproduction: «Figure de paille des Moïs (Indochine)» - se trouvait déjà dans Les Voix du silence (p. 559560), et le verdict était sans appel: «Mais ce ne sont pas les figures de paille que 
opposition au surréalisme et à Breton - à inscrire l'Afrique, et notamment la sculpture africaine, dans une relation complexe avec l'Occident. D'abord un rapport de continuité avec l'histoire de la création telle qu'il la conçoit: comme conquête de la liberté, du “droit à l'arbitraire". L'un des axes de la pensée malrucienne est en effet la conquête, par l'art, de son autonomie, et celle-ci peut s'entendre en un double sens: autonomie à l'égard des surmondes auxquels l'art, dans le passé, a cru être subordonné: le sacré (pour le surnaturel) et la beauté (pour l'irréel); autonomie à l'égard du spectacle que l'œuvre était censée représenter - et c'est bien sûr en ce dernier sens que la sculpture africaine est proprement contemporaine: elle permets aux artistes du $\mathrm{XX}^{\circ}$ siècle de découvrir en elle cette liberté ou ce droit à l'arbitraire qu'ils recherchaient et qui nous apparaît comme l'une des fins de la création artistique. Ensuite, et contradictoirement, c'est dans un rapport de discontinuité que Malraux va penser le rapport de la sculpture africaine à l'Occident, dans la mesure même où la sculpture africaine, et notamment le fétiche, constitue une mise en question du Musée Imaginaire et de la volonté d'annexion qui caractérise l'Occident - occupant ainsi une place à part et fondamentale dans les Ecrits sur l'art.

On mesure donc la distance qui sépare Malraux de la plupart des penseurs du vingtième siècle (Carl Einstein mis à part ${ }^{7}$ ) quant à la fascination qu'exercent ces objet sans nom venus d'ailleurs. De quel ailleurs? Evoquer le rôle, évidemment fondamental, de la colonisation et de son indissociable revers, la décolonisation, ne suffit pas. Car le problème reste entier: s'agit-il d'un ailleurs dans l'espace ou dans le temps, voire dans les profondeurs de la psychè? Ces primitifs, ces peuples qu'on appelle primitifs, fascinent le grand public, pour qui éloignement dans l'espace et éloignement dans le temps finissent par se confondre, et qui se presse aux expositions, notamment à l'Exposition Universelle de 1889 - avec sa partie "Exposition coloniale" - et à celle de 1900, toutes deux

\footnotetext{
nous avons ressuscitées». C'est dire que cette figure ne fait pas véritablement partie du Musée Imaginaire: elle est là comme figuration de l'éphémère, c'est-à-dire comme ce contre quoi se dresse toute création, ce contre quoi se dresse le $\mathrm{Mu}$ sée Imaginaire lui-même...

${ }^{7}$ Carl Einstein mis à part? Oui et non. Oui, bien sûr, au sens où il fut l'un des premiers à reconnaître à ces objets, aussi bien africains que océaniens, un statut d'œuvre d'art à part entière. Non, cependant, car l'essentiel pour Einstein est de souligner le traitement plastique - et non pas pictural - de l'espace et de la tridimensionalité, alors qu’il s'agit pour Malraux de déterminer la place et le rôle de ces œuvres dans le Musée Imaginaire et dans notre monde-de-l'art.
} 
* (MALRAuX, André. L'Intemporel : 261.) à Paris. Oswald Spengler (Le Déclin de l'Occident est écrit avant la guerre, mais la première partie ne fut publiée qu'en 1918) s'inscrit dans ce courant d'intérêt pour ces civilisations si différentes de la civilisation occidentale. Mais alors que le grand public, ethnocentrisme aidant, refuse de considérer ces peuples comme «civilisées», Spengler souligne l'hétérogénéité des cultures et il critique l'ethnocentrisme avec lequel, par une forme de projection, nous prétendons pouvoir comprendre ces civilisations et ressentir ce que ces étranges étrangers ressentent. Ce n'est pas que Spengler refuse cette méthode de l'empathie (Einfühlung) et l'idéal qu'elle véhicule, mais il s'applique à souligner la distance qui nous sépare de ces civilisations autres pour mieux souligner l'effort que nous avons à fournir, la tâche qui nous attend, si nous voulons vraiment essayer de ressentir ce qu' «ils» ressentaient.

Mais, précisément, l'intérêt de Malraux n'est pas là. Je serais tenté de dire, en forçant bien sûr la note, qu'il ne s'intéresse pas aux hommes, et pas beaucoup plus aux cultures: il pense, à l'encontre de Spengler et dans une affinité profonde avec le dernier Walter Benjamin, l'auteur des Thèses "sur le concept d'histoire", que l'empathie est une illusion et que nous sommes sans doute condamnés à ne rien connaitre - à ne rien pouvoir partager - des sentiments que pouvaient éprouver ces hommes appartenant à des cultures dont l'ethnologie, alors naissante, ne nous transmet qu'une vision sans doute assez éloignée de ce que pouvait être la réalité. La désinvolture avec laquelle il invoque Marcel Griaule (parenthèse et point d'interrogation), à propos de la philosophie des Dogons, est révélatrice: “ [...] nous ne soumettons pas celle [l'admiration] que nous éprouvons devant les masques kanaga à la philosophie dogon, transmise (?) par Griaule, inconnue avant la guerre de 1914 ".* A l'encontre d'une opinion très largement répandue, y compris chez les "malruciens", il faut affirmer que la diversité des cultures n'est pas ce qui est au cœur de la réflexion de Malraux, notamment dans les Ecrits sur l'art. Il le souligne lui-même lorsqu'il insiste sur la nécessité de distinguer entre ces trois ordres que sont les Etats, les cultures et les œuvres d'art - et cela relativement à la notion même d'héritage comme métamorphose:

Et d'écarter d'abord la galéjade par laquelle les cultures sont dans un pugilat permanent, à la façon des Etats. [...] Il y a des conflits politiques irréductibles; mais il est absolument faux que les conflits de 
cultures soient irréductibles par définition. Il arrive qu'ils le soient de la façon la plus grave, il arrive qu'ils ne le soient nullement.*

Cette affirmation est fondamentale car elle isole-comme une expérience cruciale - l'objet qui est au cœur de l'intérêt que les civilisations étrangères, et notamment la civilisation africaine, exercent sur lui: les œuvres d'art. ${ }^{8}$

C'était partir de bien loin, pourrait-on m'objecter, pour en arriver à ce qui nous intéresse: le rapport de Malraux et de la sculpture africaine. Et pourtant ce long préambule était nécessaire car ces œuvres d'art, qui constituent l'objet propre de sa réflexion, ne vont pas intéresser Malraux en tant qu'expression d'une culture ou d'une civilisation, dont souvent nous ne savons rien et dont parfois, ce qui est peut-être pire, nous croyons savoir quelque chose grâce à une ethnologie alors balbutiante. Malraux prend ainsi ses distances avec une vulgate hégélienne, plus ou moins mise à la mode par Taine et en tout cas encore très vivace chez lui. Si une époque ne produit pas ses œuvres d'art comme un pommier produit ses fruits ${ }^{9}$ - selon l'image même utilisée par Malraux pour dénoncer une approche matérialiste ou étroitement sociologique de la création artistique - cela vaut également pour la civilisation africaine: celle-ci - pour autant qu'on puisse parler d'elle au singulier - ne produit pas ses sculptures comme un pommier ses pommes. La notion d'expression ${ }^{10}$ n'est donc pas pertinente pour l'étude de la création artistique. Elle ne le serait - mais c'est bien contre cela que Malraux ne cesse de se battre - que si l'on confondait création et production, réduisant la première à la seconde. Le regard que porte Malraux sur les œuvres d'art, et notamment sur la sculpture africaine, est donc d'emblée un regard qui se distingue du regard ethnologique. Sommes-nous ici en présence d'un traitement qui serait propre à la sculpture africaine? Pas totalement, si l'on veut bien se souvenir de la critique adressée par Malraux à l'Université: celle-ci, comme il l'affirme explicitement dans L'Homme précaire et la littérature, ${ }^{*}$ enseigne non la littérature, mais son histoire. $\mathrm{Ce}$

\footnotetext{
${ }^{8}$ Ce sont elles que Malraux désigne lorsqu'il écrit: "et le problème qui se pose, c'est précisément de savoir ce qui assure la transcendance partielle des cultures mortes." (Ibidem: 278.)

9 "On a cru qu'une époque produit son art, comme un pommier ses pommes" (MALRAUX, A. L'Intemporel: 275.)

${ }^{10}$ Les fétiches, au même titre que les idoles, la Majesté d'Eléphanta, le Tympan de Moissac ou le Shigémori de Takanobu, "appartiennent à des arts d'accession, non à ceux que nous appelons arts d'expression.” (Ibidem: 282.)
}

* (MALRAUX, André. Postface aux Conquérants. In: Oeuvres complètes I: 273274.)
* (MALRAUX, A. L'Homme précaire et la littérature: 7 .) 
* (MALRAUX, André. La Tête d'Obsidienne: 125.)

* (MALRAuX, André. Les Voix du silence. Paris: Gallimard, 1951: 63.)

* (MALRAUX. André. Préface à "Chefs-d'œuvre de l'art primitif". In: OC, V: 1217.) qui renvoie à l'opposition, centrale chez Malraux, entre l'Université et la "secte", entre la connaissance et l'émotion: "L'enseignement de Corneille est une chose, la représentation du Cid en est une autre." 11 Or l'émotion qui caractérise notre rapport à tel texte ou telle œuvre est indépendante de notre connaissance de ses conditions de production. On retrouve donc ici, très loin semble-t-il de la sculpture africaine, le même schème qui est à l'œuvre: la contextualisation d'une œuvre tend à réduire celle-ci à l'état de produit et à ignorer en elle sa dimension de création. Le savoir - qu'il soit universitaire ou ethnologique - ne rend pas compte de notre rapport aux œuvres d'art, et c'est cette idée d'une décontextualisation des œuvres - de leur citationnalité dirait Jacques Derrida - qui est au fondement même de la notion de musée Imaginaire et du dialogue qui s'instaure en et par lui: "Les sculptures qui nous parlent, elles se parlent."* La condition de cette fraternité des œuvres entre elles et de l'émotion que nous éprouvons en leur présence est tout entière dans cette décontextualisation qui est l'une des dimensions de la métamorphose - ce qui vaut pour toute œuvre d'art survivante et pas seulement pour la seule sculpture africaine: "Toute ouvre d'art survivante est amputée, et d'abord de son temps [...] Si nous parvenions à éprouver les sentiments qu'éprouvaient les premiers spectateurs d'une statue égyptienne, d'un crucifix roman, nous ne pourrions plus laisser ceux-ci au Louvre."* Mais, dans le cas de la sculpture africaine, cette affirmation d'une décontextualisation ou d'un changement de références s'accompagne d'une critique, à peine voilée, de l'ethnologie: "Notre contact avec ces figures devra toujours moins à notre connaissance de ce qu'elles représentent, voire des mythes dogon, qu'à notre relation avec le Musée imaginaire de notre temps."*

Cette contradiction entre sentiments ou expérience originaires (ou prétendus tels) et présence au sein du Musée pose le problème de la puissance d'annexion du Musée Imaginaire, et ce problème est posé dans toute son acuité dans le cas des fétiches:

Pour que les fétiches entrassent au Musée Imaginaire avec toute leur signification, il faudrait que l'homme blanc, et non tel groupe d'artistes ou d'amateurs [...] acceptât d'élire en lui sa part de profondeur.

\footnotetext{
${ }^{11}$ Discours prononcé, le 19 mars 1966, à l'occasion de l'inauguration de la maison de la culture d'Amiens.
} 
D'élire, et non d'annexer. Il ne s'agirait plus alors de savoir quelle est la place de ces arts: car s'il ne sont plus ceux de formes chargées d'autres mondes (de formes pourtant); s'il trouvent la pleine voix de leur prédication, ils n'envahissent pas le musée: "ils le brûlent [...] Si notre civilisation entend un appel à la dépossession, elle n’a pas encore abandonné la poursuite d'une totalité qui prit tant de formes, ni surtout sa volonté d'annexion.."*

Il y a là, de toute évidence, une limite infranchissable - et c'est sans doute cette limite qu'a franchie Möllberg dans le colloque des Noyers de l'Altenburg. S'il a détruit - brûlé - son manuscrit, dont le titre même - "la civilisation comme conquête et comme destin" - était porteur d'un ethnocentrisme évident, ce qui l'a poussé à renoncer, c'est, comme il l'avoue lui-même: "Pas un concept! L'Afrique."* On pourrait dire, pour reprendre le terme même de Malraux, qu’il a "élu”, ou cru “élire”, l'Afrique. Mais l'erreur de Möllberg, comme celle de Spengler, est sans doute de n'avoir pas suffisamment été sensible à ces "formes" que constituent les œuvres auxquelles les civilisations - que ce soit la civilisation occidentale ou la civilisation africaine - ont donné naissance.

C'est donc bien en tant qu'œuvres d'art que Malraux s'intéresse à la sculpture africaine: non pas aux statues des divinités, des esprits ou des ancêtres, mais aux sculptures. Un monde de $p u$ res formes? La question est complexe et la réponse doit nécessairement l'être.

En ce sens, Malraux n'est pas seulement proche de Walter Benjamin, il annonce également certaines analyses de Jacques Derrida dans La Carte postale ou dans Marges, et notamment le concept d'itérabilité. Ce que Derrida affirmera de tout langage: l'absence de liaison substantielle entre la signification et le contexte, est d'abord vrai, pour Malraux, des œuvres d'art. L'itérabilité, qui est la capacité d'être répété, ré-itéré, fonde ce que Derrida nomme une "logique qui lie la répétition à l'altéritê" - définition qui pourrait s'appliquer à la notion malrucienne de métamorphose. Car ce qui caractérise essentiellement les œuvres d'art, c'est que celles-ci ne sont jamais prisonnières de leur sens originel, qu'elles ne naissent comme œuvres d'art - et c'est manifeste dans le cas des fétiches ou des masques - que par arrachement à ce sens, par une métamorphose.

C'est donc le refus radical de toute empathie (Einfühlung) que l'on trouve déjà chez Walter Benjamin - qui est au fondement de la notion même de Musée Imaginaire et de notre rapport aux œuvres d'art. Sur ce point, Malraux ne variera jamais et tout au plus
* (MALRAuX, André. Les Voix du silence: 541 .)

* (MALRAuX, André. Les Noyers de L'Altenburg. In: Oeuvres Complètes, II. Paris: Gallimard, 1996: 692.) 
* (MALRAUX, André. La Tête d'Obsidienne: 208-209.)

* (Ibidem: 192.)

* (MALRAuX, André. Les Voix du silence: 64 .) peut-on dire qu'il a approfondi le lien entre ce refus de toute empathie et l'universalité même du langage de l'art. Qu'on y adhère ou pas - et on peut en effet critiquer Malraux sur ce point - c'est bien là sa thèse et le fondement de son universalisme. Les cultures ne sont que des idiomes ou des dialectes, seul le langage de l'art est universel ou promesse d'universalité - en témoigne l'émotion que nous éprouvons en présence de ces formes. Là est peut-être le talon d'Achille de la pensée malrucienne: un "nous" qui se veut universel, résultat de l'histoire, une sorte de fin de l'art et de l'histoire de l'art, héritier en plus d'un sens de la conception hégélienne, mais dont nous savons - et Malraux le savait déjà - qu'il n'est sans doute lui-même qu'une figure historique et comme telle transitoire. Même s'il n'en traite nulle part explicitement, certains textes montrent que Malraux a été conscient de ce problème: "le Musée Imaginaire n’a pas envahi le monde, apporté par les conquérants: il l'a conquis parce que son émancipation de la beauté, et même de la culture, le chargeait de la promesse d'un langage universel. Le Musée Imaginaire, provisoirement, c'est l'Occident."* Ce "provisoirement», qui pourrait ne pas être dépourvu d'une certaine arrogance occidentale, est en même temps la marque d'une faille au sein même de la maitrise qui caractérise le Musée Imaginaire et l'Occident et à tout le moins d'une interrogation.

Quoi qu'il en soit de cette question de l'universel, et de l'émotion que nous éprouvons en présence de ces formes, il faut souligner qu'on ne peut - notamment dans le cas de la sculpture africaine parler de pures formes. Car ces formes, précisément, sont impures. Impures - d'une impureté magistrale pourrait-on dire - au sens où quelque chose en elles résiste à leur annexion par le Musée Imaginaire. Malraux est si conscient de l'importance de ce problème qu'il y revient plusieurs fois (sans même parler des innombrables passages consacrés aux génies réfractaires) et avec un léger tremblé dans l'écriture, comme si la saisie adéquate de l'idée se dérobait. Car Malraux affirme bien que les sculptures africaines entrent au Musée Imaginaire sans y perdre leur sauvagerie: "Bien que le ferment européen l'ait ressuscité parmi d'autres chefs-d'œuvre, il [le Shigemori de Takanobu] entre au Musée Imaginaire sans y perdre son recueillement, comme les sculptures sauvages y entrent sans y perdre leur sauvagerie", ${ }^{*}$ mais il lui arrive d'être plus précis et d'utiliser les termes d'aura* ou d'empreinte, car ce que les sculptures sauvages ne sauraient perdre, c'est l'empreinte de cette sauvagerie: "La 
métamorphose que subit le masque lorsqu'il entre au musée efface la sorcellerie, n’efface pas son empreinte: le fétiche le plus géométrique ne devient pas une sculpture cubiste."*

C'est dire que leur métamorphose - la métamorphose des "sculptures sauvages" en œuvres d'art - n'est pas seulement inachevée, elle est inachevable - et que cet inachèvement même est constitutif, du moins pour partie, de l'émotion qu'elles nous procurent, de ce que Malraux nomme leur présence. L'ontologie de l'œuvre d'art serait donc ici, dans le cas des fétiches, une hantologie (j'emprunte ce terme à Jacques Derrida): l'œuvre est hantée par le fantôme de ce qu'elle fut, de cette origine dont elle a été séparée et délivrée, mais qui revient sous forme de fantôme ou d'empreinte. Il y a là une appropriation, ${ }^{12}$ par Malraux, de la notion d'aura telle que l'entendait Walter Benjamin. Et se joue là quelque chose d'essentiel. Car c'est précisément cette trace de ce qu'elles furent, cette aura ou cette empreinte, cette forme d'échec de la métamorphose - d'une métamorphose totale, achevée, synonyme d'harmonie esthétique - qui fait de ces sculptures non seulement des œuvres exorbitantes, mais, en tant que telles, des œuvres sans lesquelles le Musée Imaginaire ne serait pas ce qu'il est et n'aurait peut-être pas été conçu. Affirmation qui peut paraître excessive, mais qui est parfaitement fondée si on accepte de lire avec rigueur les textes de Malraux. Sans doute doit-on reconnaître que cette empreinte - survivance sur le mode de l'absence de ce qui fut présent - vaut pour toutes les œuvres du surnaturel et donc pour une sculpture romane ou pour un crucifix gothique comme pour un fétiche. Mais les fétiches, par la force même dont ils sont habités, parce qu'ils touchent à "la face obscure de l'homme et du monde", à ce que Malraux répugne à appeler la magie, résistent plus que toute autre création à la réduction au seul domaine esthétique (au passage pur et simple de la valeur cultuelle à la valeur d'exposition, pour reprendre les catégories de Benjamin): "Nous pouvons dire, à la rigueur, que la magie $[. .$.$] se$ métamorphose en art; en aucun cas, que nous les confondons avec

\footnotetext{
${ }^{12}$ Je prends ce terme au sens de Derrida: lorsqu'il parle d'exappropriation, il postule qu'un texte, ou un terme, appartient en propre à un auteur mais qu'en même temps celui-ci, qui n'a pas le monopole du sens, désire en être exproprié, ce qui seul peut permettre la survie du texte. C'est toute la logique de l'itérabilité, d'une "répétition dans l'altérité", qui est ici à l'œuvre - comme dans la notion malrucienne de métamorphose. Le concept d'aura, que Malraux emprunte à Benjamin, en transformant sensiblement son sens, survit ainsi dans les Ecrits sur l'art.
}

* (MALRAUX. André. Préface à "Chefs-d'œuvre de l'art primitif". In: Oeuvres Complètes, V Paris: Gallimard, 2004: 1216.) 
une volonté exclusivement esthétique." ${ }^{13 *}$ En ce sens, je l'ai montré ailleurs, ils sont à la marge du Musée Imaginaire, ils le mettent en question, sinon en péril, et c'est précisément ce qui différencie le Musée Imaginaire d'un musée quelconque, possèderait-il en lui un "cabinet de curiosités", ou un "département de cessions".

On doit même affirmer que c'est en un double sens que la sculpture africaine est à la marge du Musée Imaginaire: au niveau anthropologique, d'abord, parce qu'elle touche à "la face nocturne de l'homme"* et du monde, qu'elle "suggère un chemin" vers l'im-

* (MALRAUX. André. Préface à "Chefs-d'œuvre de l'art primitif". In: Oeuvres Complètes, V: 1217.$)$

* (MALRAUX, André. L'Intemporel: 279.)

* (MALRAUX, André. Oeuvres Complètes, V: 938.) mémorial et "sculpte le mystère"; ${ }^{*}$ au niveau logique, ensuite, parce qu'elle met en question le Musée Imaginaire, au même titre que les dessins d'enfants, de fous, ou les ready-made de Duchamp. Et les deux niveaux se confondent si peu que Malraux les oppose parfois, ce qui est bien une façon de les distinguer, car dans le ready-made la dimension anthropologique (ou métaphysique, si l'on veut) est absente: "Mais le jury du Musée imaginaire est orienté, lui aussi, parce que la métamorphose du sèche-bouteilles en sculpture, des pierres précieuses en tableaux, est loin de nous atteindre à la profondeur où nous atteint la métamorphose des fétiches en œuvres d'art".* C'est qu'à ce second niveau se joue une interrogation qui excède de toute évidence le domaine esthétique, interrogation qui est, selon Malraux, à la fois immémoriale et contemporaine, et qui n'est autre que notre rapport au sacré. Prenant ses distances avec la définition du sacré comme "tout autre", due à Rudolf Otto, qui installerait l'homme dans un état de séparation ou de soumission absolue à l'absolu, Malraux va insister sur le rôle d'intercesseur que la sculpture africaine, précisément parce qu'elle est "le surnaturel à l'état pur", peut jouer dans notre rapport au "mystère":

La profondeur de l'art africain est moins ce qui nous échappe sans recours, l'insondable Majesté à quoi répond la prosternation, ${ }^{14}$ qu'une approche de notre élément secret et inaccessible, une communion avec lui $[\ldots]$ Le surnaturel sauvage, au contraire, suggère un chemin, fût-il menaçant; car vers l'immémorial, vers la caverne, le plus sinistre fétiche est un intercesseur. Le contraire du ciel étoi-

\footnotetext{
${ }^{13}$ Ce refus d'une réduction de la création artistique à une volonté exclusivement esthétique - refus qui fonde l'opposition à Maurice Denis - est affirmé dès Les Voix du silence: "Un grand artiste qui ne connaîtrait, outre les œuvres contemporaines, que les qualités spécifiquement plastiques des œuvres du passé, serait le type supérieur du barbare moderne: celui dont la barbarie ne se définit plus par le refus de la cité, mais par le refus de la qualité humaine».

${ }^{14}$ Comment s'étonner d'une telle position - refus de la prosternation - chez Malraux qui définit l'art comme un "anti-destin".
} 
lé. Il nous mène vers la part brumeuse du monde, à travers la nôtre. Comme le mythe [...] il capte le mystère, non en l'élucidant, mais en l'interrogeant.*

Et si cette interrogation immémoriale est à ce point contemporaine, c'est sans doute parce que notre civilisation, qui se veut héritière de toutes celles qui l'ont précédée, est en elle-même et pour elle-même une civilisation privée de toute valeur propre ordonnatrice, une civilisation qui ne se conçoit pas, ${ }^{*}$ une civilisation de l'aléatoire:" "Nous n'avons pas conquis le passé du monde malgré l'aléatoire, mais par lui [...] C'est dans la vacuité, la marge, l'attente, que les arts communient entre eux."* Dans ces formes "primordiales"* qui naissent de l'irrationnel des fétiches, on comprend que Malraux puisse écrire que nous avons à découvrir "l'obscure part de l'artiste occidental dont nous ne savons pas encore si elle s'appellera mystère ou cancer."*

Le lieu où sont étudiées ces œuvres qui se situent aux marges du Musée Imaginaire, non dans Le Surnaturel (alors même que "Les masques sauvages, quelques effigies de morts ou d'esprits, présentent sans doute les formes les plus chargées de surnaturel que nous connaissions"), * mais au chapitre IX de L'Intemporel, est révélateur: comme Malraux le précise lui-même dans une note manuscrite, ${ }^{16}$ ces derniers chapitres sont consacrés aux arts qui mettent en question le Musée Imaginaire. Ce n'est donc pas un hasard si Malraux accorde une telle place - dans tous les sens du terme - à la sculpture africaine, et s'il joue parfois avec elle comme on joue avec le feu. C'est qu'il a très tôt, dès Les Voix du silence, pris conscience (comme Picasso lui-même, si l'on en croit La Tête d'Obsidienne) que ces sculptures n'étaient pas des sculptures comme les autres. Il a pris conscience de la force qui les habitait encore et qui en un sens les habiterait toujours, à tel point - on l'a vu - qu'il souligne que s'ils retrouvaient cette force (cette force elle-même et non pas son empreinte ou sa trace au sein de la forme quaccueille le $\mathrm{Mu}$ sée) les fétiches pourraient bien brûler le Musée. Mais aussi que ces sculptures, ou certaines d'entre elles, étaient par leur style même, aux marges du Musée Imaginaire: “Ce style met au défi l’idée

\footnotetext{
${ }^{15}$ C'est dans cette civilisation que vit l'homme précaire: «car le regard posé sur la mort par l'homme de l'aléatoire, est celui d'un Socrate sans dieux" (Oeuvres Complètes, VI: 924).

16 "Le sujet général à partir du ch. VII est la mise en question du M.I du XX" (Oeuvres Complètes, V: 1483.)
}

* (MALRAUX, André. L'Intemporel: 279.)

* (MALRAuX, André. Oeuvres Complètes, VI. Paris: Gallimard, 2010: 917.)

* (MALRAuX, André. L'Homme précaire et la lit térature: 321.$)$

* (MALRAuX, André. L'Intemporel: 311.$)$

* (Ibidem: 304.)

* (lbidem: 256.) 
de style."* Ce regard malrucien sur la sculpture africaine éclaire la totalité des Ecrits sur l'art, puisque c'est à ce niveau, et à ce niveau seulement, que se joue véritablement l'ouverture du Musée Imaginaire. Cette ouverture ne serait qu'un mot - un mot vide de tout contenu - si elle devait se limiter à accueillir les œuvres qui correspondent à la conception de l'art qui est ou a été la nôtre, que nous avons héritée de notre tradition - et d'abord de l'Antiquité et de la Renaissance. Ce qui est en jeu avec l'art africain - et cela n'apparaît pas seulement dans les Ecrits sur l'art mais également dans Le Miroir des limbes, dans le dialogue avec Senghor - c'est le caractère universel ou non de l'esthétique classique et du primat du Beau. Il va de soi que le Musée Imaginaire suppose la mise en cause de cette prétendue universalité, comme de la doctrine de l'imitation, et que la sculpture africaine n'est pas étrangère à cette mise en cause: "Lart moderne eût-il suffit à nous opérer de la s? Le fétiche, sculpture dérisoire parce que l'art avait pour objet l'imitation, l'était aussi lorsque l'homme devait pratiquer une religion raison-

* (MALRAUX, André. Oeuvres Complètes, V: 894.)

*(MALRAUX, André. La Tête d'Obsidienne: 208.) nable, et vaincre les superstitions."* Par ce regard singulier porté sur l'art africain, Malraux est résolument contemporain, et se sépare explicitement d'un Paul Valéry, incarnation, à ses yeux, d'une esthétique qui reste classique:

L'esprit n'aime pas non plus les esprits [...] L'esprit ne suffit pas à rendre intelligible l'opération qui créa tour à tour la frise du Parthénon, le Shigémori et le masque Dogon; les arts que l'homme gouverne peut-être, et ceux qu'il ne gouverne pas. Moins encore, l'opération par laquelle ces œuvres nous parlent toutes trois.*

Mais on pourrait également dire que ce qui est en jeu avec l'art africain, c'est la capacité de l'Occident de se dépasser lui-même, d'accueillir - dans ce Musée Imaginaire qui est le sien mais qui le déborde de toutes parts - les œuvres les plus éloignées de lui, les plus différentes et en un sens les plus opposées.

Reste que, et c'est tout le problème, l'Occident n'accueille ces œuvres que pour autant qu'il leur reconnait ou leur accorde le statut d'œuvres d'art. Cette métamorphose, nous en mesurons aujourd'hui l'importance, notamment avec l'ouverture et le succès du Musée du Quai Branly à Paris, mais elle n’est pas dépourvue d'ambiguïté. Est-elle une forme déguisée et infiniment subtile d'ethnocentrisme: l'Occident imposant le regard qu'il faut porter sur ces objets? Cette question, pour gênante qu'elle soit, mérite 
d'être posée, même si la réponse n'est pas simple et si la question est sans doute condamnée à demeurer ouverte.

Au terme de cette brève approche des rapports de Malraux et de l'art africain, comment conclure? Peut-être en soulignant qu'il serait possible, mais vain, de tenter de faire l'inventaire de tous les textes dans lesquels Malraux traite de la sculpture africaine. Cela serait certes possible. Il faudrait procéder méthodiquement: les grands textes d'abord, parties ou chapitres de certains livres, les passages plus brefs ensuite, minuscules blocs parfois insérés dans un contexte qui n'est pas celui de l'art africain. Fraternité des œuvres oblige: de tels passages montreraient, en quelque sorte en acte, la méthode de Malraux et l'étroite corrélation entre méthode et doctrine puisque c'est le contenu même de sa pensée qui conduit Malraux à ces rapprochements dans l'espace et le temps, ces télescopages qui lui ont été si souvent reprochés. Mais cela serait vain, parce que cela donnerait une fausse image de l'importance de l'Afrique dans les Ecrits sur l'art. Car celle-ci n'est pas présente dans les seuls passages dans lesquels Malraux l'invoque: elle est au fondement même de ces écrits et les traverse de part en part, à tel point que sans elle - et notamment sans la sculpture africaine - les Ecrits sur l'art eux-mêmes n'existeraient sans doute pas et les concepts qui sont au cœur de ces écrits (Musée Imaginaire, annexion, métamorphose, surnaturel) n'auraient pu être forgés. Pour ne rien dire des concepts en apparence, mais en apparence seulement, plus indépendants de l'art africain: beauté, ressemblance, imitation, liberté, arbitraire. ${ }^{17}$ Pour ne rien dire non plus des concepts qui sont si étroitement liés à l'art africain qu'ils se confondent presque avec lui: sacré, empreinte, sauvagerie, arts d'accession opposés aux arts d'expression, et bien sûr les notions fondamentales de clôture et d'ouverture des mondes de l'art: "Les œuvres d'art étaient religieuses, belles (idéalisées) ou raffinées. Dans ce monde clos, comment un masque nègre fût-il devenu une œuvre d'art? Aussi ne l'est-il devenu que lorsque ce monde cessa d'être clos."* Car la sculpture africaine, comme toute œuvre mais plus que toute œuvre, n'est pas seulement métamorphosée, accueillie ou annexée par le Musée Imaginaire, elle transforme et métamorphose celui-ci: "Pour que le masque Kanaga se métamorphose en œuvre d'art, il faut

\footnotetext{
${ }^{17}$ Le rôle de l'arbitraire dans la sculpture africaine et l'importance que cette notion a pu jouer - précisément par la médiation de cette sculpture - dans l'histoire de l'art telle que l'entend Malraux mériterait, à lui seul, une étude séparée.
}

* (MAlRAuX, André. L'Intemporel: 246 .) 
que se métamorphose insensiblement le domaine de références qui va l'annexer. Et il y contribue."* Notons qu'il y a là, dans le texte imprimé, un double alinéa, qui isole l'affirmation «Et il y contribue»: comme si Malraux, anticipant le débat contemporain entre structure et événement - débat qui vise, contre certains excès, à rappeler l'importance de l'événement - avait voulu isoler l'événement et souligner son importance dans la structure. Et pour souligner la rupture que la découverte de la sculpture africaine instaure dans l'histoire de l'art, Malraux affirme explicitement que "les problèmes [de la création] différés cessent de l'être lorsque l'art nègre entre en jeu".* Si le début du siècle - avec Guillaume Apollinaire et Carl Einstein - avait œuvré pour faire admettre les œuvres africaines comme des œuvres d'art à part entière, il revient à Malraux d'en avoir fait l'élément dynamique - le négatif - de notre monde de l'art et du Musée Imaginaire.

Le dernier mot sera laissé à Malraux pour souligner l'importance de cette notion de "langue" propre aux fétiches qu'il leur reconnaît, et qui permet de récuser l'assimilation si souvent faite entre la sculpture africaine et l'archaïque ou le primitif, voire le préhistorique, la réduction de la première aux seconds: "Le fétiche ne balbutie pas la langue des formes humaines, il parle la sienne: les plus proches deviendront ses traductrices."*

\section{Jean-Pierre Zarader}

Professor "agrégé" de filosofia. Autor de Malraux ou la pensée de l'art (Paris: Ellipses, 1998) e de um Vocabulaire de Malraux (Paris: Ellipses, 2001). Publicou ainda: Philosophie et cinéma (Paris: Ellipses, 1997), Robinson philosophe: Vendredi ou la vie sauvage de Michel Tournier, un parcours philosophique - suivi de "Le philosophe aux images", um entretien avec Michel Tournier (Paris: Ellipses, 1999), Matrix, Machine Philosophique (em colaboração com Alain Badiou, T. Benatouil, E. During, P. Maniglier, D. Rabouin, Paris: Ellipses, 2003) e Lectures de Sartre (em colaboração com Philippe Cabestan, Paris: Ellipses, 2011).

Mots-clés: Malraux; Picasso; sculpture africaine; musée.

\section{Résumé}

Qu'advient-il du sacré dans notre monde profane? Partant de la scène mythique dans laquelle Picasso, au musée du Trocadéro, ressent le caractère magique des masques, Malraux s'interroge sur la relation agonistique qui existe entre formes et forces. Contre la vulgate qui 
tend à voir dans l'esthétique une relève du sacré - ce qui conduirait à une clôture dans l'immanence -, peut-être doit-on tout à la fois refuser l'illusion d'une empathie et affirmer le caractère inachevable de la métamorphose des fétiches en œuvres d'art - inachèvement que Malraux désigne par les termes d'empreinte ou d'aura.

\section{Resumo}

Que acontece com o sagrado em nosso mundo profano? Partindo da cena mítica na qual Picasso, no museu do Trocadéro, sente o caráter mágico das máscaras, Malraux interroga-se sobre a relação agônica que existe entre formas e forças. Contra a vulgata que tende a ver na estética um sucedâneo do sagrado - o que conduziria a um fechamento na imanência - , talvez devêssemos, ao mesmo tempo, recusar a ilusão de uma empatia $e$ afirmar o caráter inacabado da metamorfose dos fetiches em obras de arte - inacabamento que Malraux designa pelos termos de empreinte (marca) ou de aura (aura).

\section{Abstract}

What happens with the sacred in our profane world? Inspired by the mythical scene in which Picasso, at the Trocadéro museum, feels the magic character of the masks, Malraux wonders about the agonic relationship between forms and forces. Against the common perception which tends to see in the aesthetic a substitute for the sacred - which would lead to a closure in immanence -, perhaps we should, at the same time, refuse the illusion of empathy and affirm the unfinished character of the metamorphosis of fetishes into works of art - incompleteness designed by Malraux by the terms of empreinte (imprint) or aura (aura).
Palavras-chave: Malraux; Picasso; escultura africana; museu.

Keywords: Malraux; Picasso; african sculpture; museum. 